\title{
Tachistoscopic display using color-lookup-table display devices
}

\author{
PETER DIXON \\ University of Alberta, Edmonton, Alberta, Canada
}

\begin{abstract}
In this article, I describe a method for presenting multiple bit-mapped images on video displays under precise temporal control. The method makes use of the color-table animation technique that can be used with color-lookup-table display devices. The principal challenge in applying this technique to the display of multiple stimuli lies in dealing efficiently with the problem of overlapping displays. The solution described here is to allocate separate bit slices of screen memory to stimuli that overlap and to allocate sequential color-table indices to stimuli that are disjoint. Software is described that automatically computes the location of a stimulus on the screen, allocates color indices appropriately, transfers bit images to screen memory efficiently, and manipulates the color lookup table to present a given stimulus to the viewer. These operations are handled transparently; the user simply draws the stimuli and then turns them on or off under program control.
\end{abstract}

A considerable proportion of the research in cognition and perception depends on presenting visual displays under precise temporal control. For example, researchers interested in reaction time measurement need to know the exact time at which a visual stimulus is presented, those concerned with visual information processing may need to present a stimulus for a fixed duration followed at some interval by a masking stimulus, and researchers interested in apparent motion may need to present several displays separated by specific stimulus-onset asynchronies. I use the term tachistoscopic display to refer to the general problem of presenting a number of visual stimuli successively with accurate timing, even though in many cases a given stimulus might be presented for relatively long periods of time. In the present article, I describe a general procedure for generating such tachistoscopic displays under a wide range of conditions using commonly available microcomputer hardware. The procedure makes use of a technique known as color-table animation and can be implemented on any computer that uses color-lookup-table display devices. Examples of such computers include the Macintosh II family of computers, Commodore Amiga computers, and IBM PC computers using VGA and EGA video boards. Thus, the technique has wide applicability. In this article, I first describe the color-table animation technique and how it can be used for tachistoscopic display. Next, I present an efficient solution to the problem of using color-table animation for successive stimuli that

\footnotetext{
This research was supported by a grant from the Natural Sciences and Engineering Research Council of Canada. The author wishes to thank John Henderson and Gary Finley for comments on an earlier draft of this report. Source code (in Lightspeed Object Pascal), running example programs, and further details of the software are available from the author; software requests should be accompanied by a blank disk. Correspondence should be addressed to Peter Dixon, Department of Psychology, University of Alberta, Edmonton, Alberta T6G 2E9, Canada.
}

overlap on the screen. Finally, I describe a particular implementation of this algorithm on the Macintosh II.

\section{The Color-Table Animation Technique}

Presenting stimuli tachistoscopically using the rastertype display devices commonly found on most microcomputers has represented a challenge to psychological researchers. One simple solution is to use software-controlled video blanking (e.g., Heathcote, 1988; Reed, 1979). In this case, the screen is first turned off, the stimulus is drawn on the screen, and then the screen is turned on and off to present the stimulus for the required display duration. A related solution is to use software to switch among the multiple screen buffers that are available on some computers. Other techniques involve transferring bit images to the screen just ahead of the video raster scan, so that the entire screen is changed in one refresh cycle (e.g., Costin, 1988). While these techniques have had some success, they do not provide a completely general solution to the problem of how to perform tachistoscopic display. For example, using display blanking, one can present only a single display under precise temporal control. Multiple screen buffers overcome this limitation to some extent, but most computers have only a small number of different screen buffers. For example, the Apple II has four buffers, and the early Macintosh models have two. Drawing the display ahead of the raster scan requires an increasingly fast processor to do the drawing as the size and depth of the display screen increase. For example, the technique used by Costin will work on a "vintage" Macintosh, but not on a Macintosh II with a large color display. If the stimulus consists of text, many of these problems can be ameliorated by using display hardware that generates characters based on ASCII codes in memory (e.g., Finley, 1989; Graves \& Bradley, 1987). However, with this approach, one gives up many of the capabilities of bit-mapped displays, such as the ability to present text 
in a large number of different fonts and styles, the ability to freely mix text and graphics, precise control over the position of the stimuli on the screen, and so on.

Color-lookup display devices provide a powerful and general solution to this problem. A color-lookup display device is designed to present a large variety of different colors on a video monitor using a relatively small amount of computer memory. Each pixel on the screen is mapped onto a set of bits in the computer's memory. Typically, these bits reside in a designated portion of the computer's address space known as screen memory, but this need not be the case. However, the set of bits used for each pixel does not determine directly the color shown on the screen. Instead, the hardware interprets the bits as an index into a color-lookup table that contains colors for all possible indices; the color shown on the video screen is determined by the entries in this table. Generally, the number of entries in the table is much smaller than the number of possible colors that could potentially be shown on the screen. For example, on the Macintosh II color video board, there are 256 entries in the color-lookup table, but each entry in the table can designate one of more than $10^{14}$ different colors. ${ }^{1}$ A main advantage of this kind of display hardware is that there is a large range in the number of potential colors that can be shown even though the amount of screen memory is relatively small. The main limitation of the approach is that the number of different colors that can be shown simultaneously is limited to the size of the color-lookup table. Thus, the users of the device are given control of the color-lookup table so that they can customize the palette of available colors to their needs.

With this kind of hardware, the color-table animation technique can be used to present monochromatic stimuli under precise temporal control. The approach is illustrated in Figure 1. Suppose that screen memory initially contains only zeros and that the index zero is associated with white in the color-lookup table; under these circumstances, the video display will simply show a blank white field (see Figure 1a). The first step in using color-table animation is to select an index that is not present anywhere in screen memory and to associate that index with the background color of the screen. For example, in Figure $1 \mathrm{~b}$, the color associated with the index 1 is set to white. The stimulus is then drawn in screen memory using this index. For example, in Figure 1c, all of the pixels in screen memory corresponding to a rectangular area are given the index 1 . However, the stimulus will be invisible to anyone looking at the video display, because the pixels on the screen will be plotted in exactly the same color as the background. In order to make the stimulus visible, the color associated with the index is changed from the background color to some contrasting color. For example, in Figure 1d, the rectangle is presented by changing the entry in the table for index 1 from white to black. When the stimulus is to be removed from the screen, the color-table entry would be changed back to white again. Because changing an entry in the color-lookup table requires modifying only a few bytes in memory, stimulus
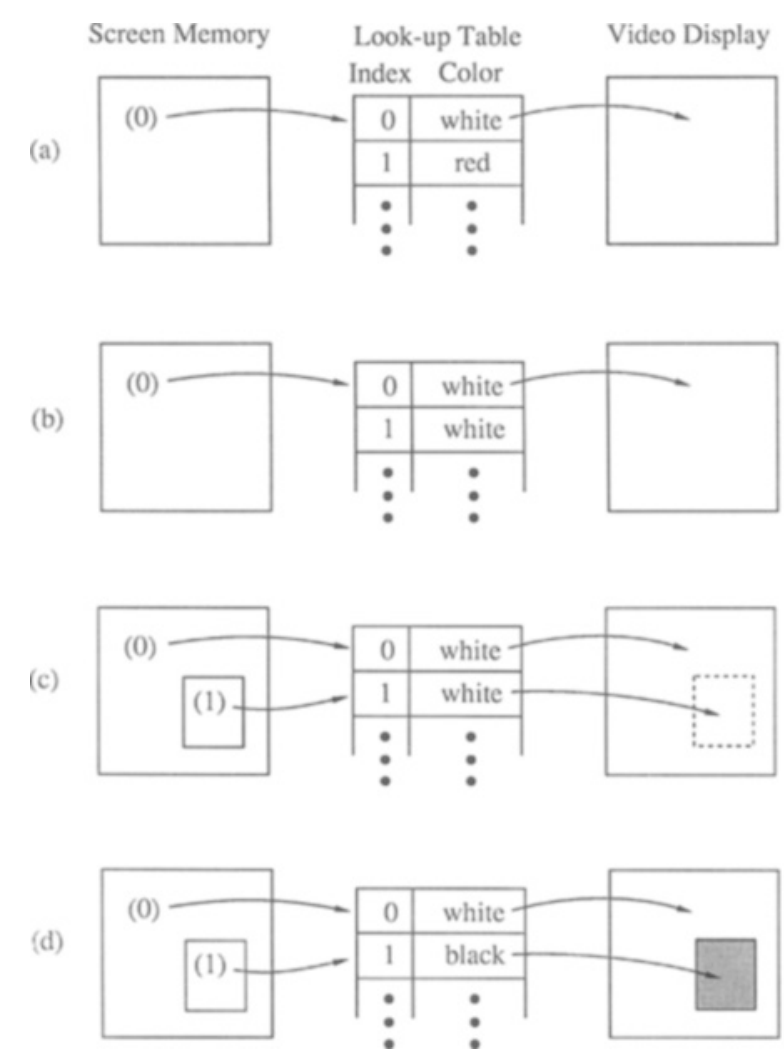

Figure 1. Steps in using color-table animation for tachistoscopic presentation of stimuli (see text for explanation).

onset and offset is rapid and generally can be accomplished during the video blanking interval. This means that the visual display can be changed, for all intents and purposes, instantaneously.

The promise of this approach is that a very large number of possible displays can be shown in any temporal configuration. For example, if the screen memory for the display device is 8 bits deep (i.e., 1 byte per pixel), there would be 256 entries in the color-lookup table (one for each possible pixel value); if one index is used for the background, the remaining 255 indices potentially could be used for tachistoscopic display. Thus, in principle, up to 255 different stimuli could be drawn on the screen first, and then each of these stimuli could be shown individually under program control.

\section{The Problem of Overlapping Displays}

The flaw in this reasoning concerns overlap among displays. In order to display a particular stimulus using colortable animation, all of the pixels in screen memory that make up the stimulus must contain a particular index into the color table. Thus, if another stimulus to be presented later uses a pixel that also appeared in the first stimulus, there will be a conflict: If the pixel contains the first index, there will be a hole in the second stimulus; however, if the pixel contains the second index, there will be a hole 
in the first stimulus. The solution to this problem is to use indices that explicitly code where the stimuli overlap. For example, if Stimulus 1 is drawn with index $n$ and Stimulus 2 is drawn with index $m$, then one would allocate a third index, $p$, that would be used for all of the pixels that occur in both stimuli. Then, when Stimulus 1 was to be shown, the colors associated with both $n$ and $p$ would be changed in the color table; when Stimulus 2 was to be shown, the colors associated with $m$ and $p$ would be changed. Unfortunately, calculating which pixels occur in a stimulus by itself and which also occur in overlapping stimuli can be computationally cumbersome. For example, one approach would be to first draw a stimulus in an offscreen buffer. This image would then be differenced with the second display to find those pixels that are in Display 1 only; these pixels would be transferred to screen memory using index $n$. The image would then be redrawn offscreen and intersected with the second display; these pixels would constitute the overlap and would be drawn on the screen using index $p$. This kind of algorithm is inefficient in that it requires six drawing operations to put one display on the screen. Moreover, the computation required to calculate overlaps explicitly would grow exponentially with the number of displays that need to be in screen memory simultaneously.

Another way to solve the overlap problem is to calculate the overlaps implicitly. In this approach, each stimulus to be shown is allocated a bit slice from screen memory. For example, Stimulus A might be given bit 0 , Stimulus B might be given bit 1 , and so on. Then, to draw any of the stimuli, one would simply add the index corresponding to the given bit slice to the index that already existed in screen memory. This is illustrated in Figure 2. In this example, Stimulus $A$ would use 1 as its index (i.e., bit 0 ), Stimulus $B$ would use 2 as its index (i.e., bit 1 ), and any pixel that occurs in both stimuli would end up with an index of 3 (i.e., both bits 0 and 1). Turning on a given stimulus display would consist of changing the colors associated with all of the animating indices that have the appropriate bit set. In the present example, indices 1 and 3 would be changed for Stimulus $A$, and indices 2 and 3 would be changed for Stimulus B. I will refer to this procedure as the bit-slice method. In general, the

Indices in Screen Memory

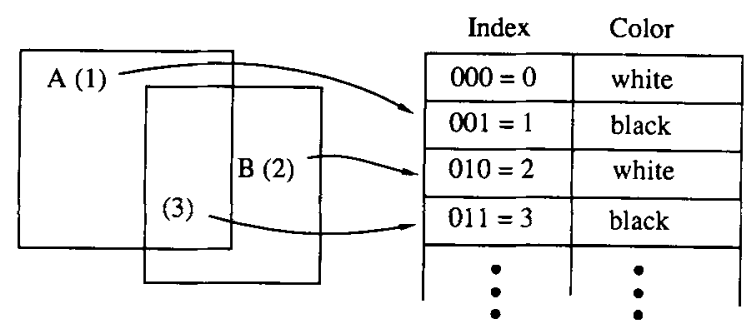

Figure 2. Regions of screen memory with color-lookup indices (indicated in parentheses) allocated according to the bit-slice method. The color-table entries have been set to show Stimulus $A$. bit-slice method will be more efficient than calculating overlaps explicitly, because each display is only drawn once. Moreover, it works equally well for any number of overlapping displays. For example, if there were three stimuli using bits 0,1 , and 2 , respectively, then the overlap between Stimuli A and B would be index 3, the overlap between Stimuli $A$ and $C$ would be the index 5 , the overlap between Stimuli B and $C$ would be the index 6 , and any pixels in all three of the stimuli would have the index 7 .

The limitation of the bit-slice method is that the number of concurrent displays that can be represented in screen memory simultaneously is limited to the number of bits used for each pixel. Although this may be adequate for many kinds of experiments, it would seem to underutilize the power of color-table animation when the number of displays that actually overlap is small. For example, suppose one were interested in measuring reading time using the moving-window paradigm (e.g., Dixon, 1984; Just, Carpenter, \& Wooley, 1982). In this task, a page of text is presented on the screen; however, all of the characters are initially replaced with Xs. To read the text, the subject presses a button and the Xs for the first word are replaced with the correct letters. When the button is pressed a second time, the first word is changed back to the mask of Xs and the second word is shown. In this way, each buttonpress causes a succeeding word to be presented on the screen, and the interpress latencies can be used as a measure of the reading time for each word. Stimuli in this paradigm would be difficult to present using the bit-slice method. For example, if the passage being read were 120 words long, there would be 121 different displays that would have to be represented in screen memory at the same time: The first display would be shown before the subject presses the button and would consist of just the Xs; the second display would be shown when the button is pressed the first time and would show the first word with all of the other words masked; the third display would be shown when the button is pressed the second time and would show only the second word, and so on. If a separate bit slice were used for each of these displays, 121 bits per pixel would be required. Display hardware with this kind of capability is unlikely to be available in the near future.

This limitation of the bit-slice method is frustrating because the paradigm can be implemented easily using only 9 bits per pixel if overlaps are computed explicitly. One approach would be to allocate three indices for each word: one for the word itself, one for the Xs that mask that word, and one for the overlap between the word and its masking Xs. For example, indices 1-120 could be used for the pixels that only occur, respectively, in words $1-120$; indices 121-240 could be used for pixels that only occur in the corresponding masking Xs; and indices 241-360 could be used for pixels that occur both in a word and in its mask. Then, in order to display the initial screen of Xs, one would set the color values for indices 121-360 to black. In order to show only the first word, the color value for index 1 would be changed to black, the color 
value for index 121 would be changed to white, and the color value for index 241 would be left as black; succeeding words would be shown in a similar fashion. In this method, the number of different displays that can be shown is much larger than the number of bits per pixel. Explicit calculation of overlaps is more efficient in this case because the moving-window paradigm uses a large number of different displays on a given trial, but the number of displays that actually overlap is small. The bit-slice method is inefficient in this situation, because it allows for the possibility that each display potentially overlaps with every other possible display and allocates indices accordingly. Thus, what would seem to be needed is an algorithm that can handle multiple overlapping displays in a computationally efficient manner without squandering color-lookup indices on potential overlaps that do not actually occur.

The approach I have taken to this problem is to use information about where on the screen a given stimulus is located in order to decide how to allocate indices. When stimuli overlap on the screen, the logic of the bit-slice method is used to find indices for each of the stimuli and their overlapping pixels. But when stimuli occupy disjoint regions of the display screen, indices are allocated in numerical order, giving a much larger number of possible stimuli that can be encoded in screen memory simultaneously. For example, suppose that the region occupied by each stimulus is delimited by a rectangle in screen coordinates and that stimuli are drawn on the screen successively. When a new stimulus is to be drawn, the algorithm for assigning an index to that stimulus is as follows. First, a list of all of the overlapping displays that are already drawn on the screen is calculated by comparing the given stimulus rectangle with all of the other stimulus rectangles. Using the logic of the bit-slice method, the index for the new display cannot use any of the bits in any of the indices for these overlapping displays. That is, the index used for the new display should produce a result of 0 when it is ANDed with the indices used for overlapping displays. In addition, the index allocated to the new display cannot be identical to any index used anywhere on the screen, even if the displays do not overlap. Finally, new indices will also be created implicitly for the intersections between the new stimulus and its overlapping displays; these should not be indices that are already in use elsewhere on the screen. In order to find a new index, all of the possible indices are scanned sequentially to find one that meets these constraints.

The algorithm is illustrated in Figure 3. In this example, three stimuli are already present in screen memory, and occupy regions A, B, and C. Assume that these stimuli were drawn on the screen with the indices 1,4 , and 6 . The region formed by the intersection of Stimuli A and $B$ ends up with an index equal to the sum of the respective indices, 5 . Now suppose that a new stimulus, $X$, is to be drawn. Because it overlaps with region $B$, its index cannot have bits set that are set in B's index, namely bit 2 . Possible indices that do not have bit 2 set are $1,2,3,8$,

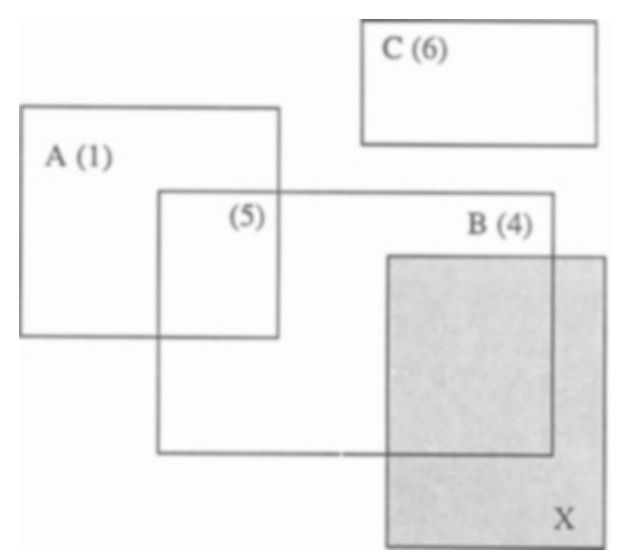

Figure 3. Regions of screen memory with color-lookup-table indices indicated in parentheses (see text for explanation).

9, and so on. Index 1 cannot be used for $X$ because it already is used for Stimulus A. If 2 were used for the index, the index used for the overlapping region $X \cap B$ would be 6, and that index is already used for Stimulus $C$. Thus, 2 would be rejected as well. An index of 3 would be considered next. In this case, the index for the overlapping region would be 7 , which is unused. Thus, 3 would be selected as the index for the new stimulus. Using the logic of the bit-slice method, the new stimulus would be drawn in screen memory by adding this index to the value already present at each pixel in the stimulus. (This has the effect of setting any pixel that occurs both in $X$ and $B$ to the index 7 , and this fact must be retained so that the correct color-table entries are changed when either X or B is actually presented.)

This algorithm represents a compromise between the bit-slice method and the explicit calculation of overlaps. For example, the moving-window paradigm described above would require 121 bits per pixel using the bit-slice method and 9 bits per pixel if the overlaps were calculated explicitly. Simulation results indicate that the present algorithm would require 10 bits per pixel. Although this is slightly more than that required by the explicit overlap method, this disadvantage is offset by the greater efficiency in drawing overlapping stimuli on the screen. In principle, the algorithm allocates indices more or less efficiently depending on the order in which stimuli are drawn on the screen. For example, it would seem most efficient to draw all of the overlapping stimuli first and then draw all of the disjoint stimuli. The disjoint stimuli then could use indices that represent gaps left by the allocation of distinct bit slices to the overlapping stimuli. However, my impression from working through a few examples is that differences in efficiency due to drawing order are not large; regardless of order, the algorithm seems to succeed in generating a reasonable compromise between the bit-slice method and the sequential allocation of indices that would occur with disjoint stimuli. For example, the moving-window paradigm requires 10 bits 
per pixel both when all of the words are drawn first followed by the masks and when words and their overlapping masks are drawn one after the other.

The usefulness of this approach depends critically on being able to identify relatively small regions of the screen in which a given stimulus will appear. In the limit, if each stimulus is assumed to occupy the entire screen, the algorithm will simply allocate a bit slice to each stimulus. Of course, this would be inefficient if the stimulus actually takes up a much smaller area. In the present implementation of the algorithm, this problem is circumvented by letting the software automatically keep track of where on the screen a particular stimulus is drawn. Thus, when one is constructing the stimulus displays for a particular experiment, one is freed from the laborious task of deciding which stimulus overlaps with which other stimulus.

\section{Implementation}

The display algorithm I have described has been implemented on the Macintosh II family of computers in Lightspeed Object Pascal. The software defines two types of objects: screens and displays. (In procedurally oriented languages, these would be defined as general data structures-for example, records in Pascal and structures in C.) Typically, the calling program would allocate one screen object and some number of display objects associated with that screen. Routines associated with these objects are defined for drawing operations and displaying operations. To draw a display, the user first allocates and initializes a display object. The display object contains an offscreen bit image for the stimulus to be drawn. The bit image is 1 bit deep-that is, each pixel in the display can be either on or off. To draw a stimulus, the display object is first opened, a sequence of drawing operations are performed, and the display is then closed. Opening the display erases any bits on the screen that belonged to the display previously and deallocates the index associated with the display. While drawing is taking place, the software automatically keeps track of which areas of the screen will be affected and collects these areas together into a display region. When the display object is closed, this display region is compared with the display regions of stimuli that are already on the screen to find the indices used by overlapping displays. On the basis of these overlaps, a new index is allocated to the display and the display is copied to screen memory using that index.

After all of the displays for a trial have been prepared in this way, the user simply turns on or off each stimulus as required. Two methods are provided for turning a display on or off. The "ready" method simply adds the relevant indices to a list of changes to be made to the colorlookup table. The "show" routine adds the indices to the change list and then actually makes these changes during the video blanking interval. These two change methods allow the user to make several display changes at the same time. For example, suppose one wanted to present a target stimulus immediately followed by a pattern mask with no intervening interstimulus interval. One would first "ready" the offset of the stimulus and then "show" the onset of the mask. This would result in the stimulus offset and mask onset being simultaneous as desired.

A central problem in implementing the present display algorithm is finding the indices that are on the screen in the region occupied by a new, to-be-added, stimulus. These indices are not simply the indices used by overlapping displays, because some of these displays may themselves overlap and the resulting intersection regions may or may not be in the region occupied by the new stimulus. To compute these indices efficiently, the software maintains a binary tree of all display regions and all possible intersections among these regions. Each node in the tree specifies a region on the screen and the index associated with that region. For example, the tree representing the screen shown in Figure 3 would consist of four nodes designating $A, B, C$, and $A \cap B$ along with the indices $1,4,6$, and 5 . When a new stimulus is added to the screen, the display region for that stimulus is compared with the regions associated with each of these nodes, and a new node is created for all nonempty intersections. In Figure 3, nodes would be created for $\mathrm{X}$ and $\mathrm{X} \cap \mathrm{B}$. The nodes created in this way constitute the overlap regions for the new stimulus and are used to determine which indices are possible for that stimulus. This tree is also used in finding the color-table entries that should be changed when a stimulus is to be turned on or off.

Several aspects of the Macintosh system make implementation of this algorithm straightforward. First, the system defines efficient routines for manipulating regions. Regions in the Macintosh drawing environment are data structures that define an arbitrary, potentially nonrectangular, shape and are ideally suited for describing the display region occupied by each stimulus. System primitives can thus be used to quickly decide, for example, whether two such regions overlap. In addition, display regions also make transferring images to the screen efficient: The system routine for copying bit images allows the region to be used as a mask so that only the bits that are actually in the image are copied.

Second, the Macintosh system allows the programmer to customize basic drawing operations. In the present implementation, all of these drawing operations are enhanced so that the area in which drawing occurs is automatically added to the display region for the current stimulus. Thus, in creating a program to draw a given stimulus on the screen, one simply uses the appropriate drawing routines in the usual way, and the software transparently keeps track of which areas need to be added to the current display region.

Finally, the Macintosh toolbox provides routines for performing color-table animation. These routines allow one to reserve a color-table index for animation and to change the color associated with that index in the color table. Generally, the index used to perform the animation is determined by the system so as to be maximally compatible with other software that might be running at 
the same time. However, recent releases of the system software allow one to use animation indices specified explicitly by the programmer (as is required in the current application). The system then takes care of ensuring that no other drawing is carried out on the screen using that index. The net effect is that the present display routines work correctly even when the stimulus display area crosses several physical display devices and generate a minimum of interference with other software that might draw on those devices concurrently.

The display software is intended to operate on any Macintosh running 32-bit color Quickdraw and has been tested on Macintosh II, IIcx, IIsi, and IIfx, using a variety of different Apple video boards. In principle, the software requires video display hardware that uses colorlookup tables; the number of indices that can be used for animation depends on the number of bits used in screen memory. If there are $n$ bits, there are $2^{n}$ possible indices; however, two of these must be reserved for black and white, leaving $2^{n}-2$ indices for animation. Apple also markets so-called "direct" color video boards that allow users to specify colors directly in screen memory without using a color-lookup table. The present software will work with these boards only if they are set to their (8-bit) colorlookup mode using the Macintosh control panel. At the present time, the software does not run correctly when the computer has an Apple graphics accelerator board $(8 \cdot 24 \mathrm{gc})$ installed and the accelerator is turned on.

\section{Extensions}

One extension to the present approach would be to store display images symbolically rather than as bit images. This method can be accomplished in a straightforward manner using the Macintosh construct of pictures. A picture is essentially a list of drawing commands that can be executed to create a given stimulus. Pictures are created by first opening the picture, executing the drawing commands, and then closing the picture; Quickdraw remembers the commands executed and can then redraw the picture whenever desired. In the present software, using pictures instead of bit images for displays would simply require that a picture be opened whenever a given display is opened and then that the picture be closed and retained whenever the display is closed. Retaining the displays in a symbolic form may or may not be more efficient than retaining bit images. Large simple stimuli will be processed more efficiently symbolically. For example, a large circle drawn on the screen would have a simple symbolic description and could be drawn quickly, whereas transferring all the bits involved would be relatively timeconsuming. On the other hand, small stimuli that require many drawing commands to create would probably be better processed as bit images.

Although the present algorithm and implementation were designed to deal with 1-bit monochromatic displays, it can be extended to deal with color or extensive gray scale. The simplest change would be to use a color or shade of gray other than black to present a stimulus on the screen. At present, each display object contains a variable indicating the color that the given stimulus is to be presented in; this is the color value that is stored in the color table when the display is turned on. Initially, this value is set to black, but it can be changed to any other color by the user. For example, one might use a level of gray so that stimuli that are presented at different brief durations would appear equally dark. A chromatic color could be chosen as well. The limitation of the present implementation is that each stimulus can have only one such color. To present simple multicolored stimuli, one could create several distinct displays, one for each of the colors in the stimulus, and then turn all of these displays on or off simultaneously. To keep this process from becoming awkward as the number of colors in a stimulus increases, the software should group all of the color-specific displays used for a given stimulus together and open and close them transparently as the calling program changes drawing colors. The number of multicolored displays that can be drawn on the screen concurrently using this technique is likely to depend critically on how much overlap there is among the colors used in different stimuli. The worst case is one in which every color in a display overlaps with almost every color used in every other display. Under such circumstances, one would be limited to no more than two 16-color stimuli or four 4-color stimuli. More colors and stimuli would be possible if the overlap was less severe.

\section{Conclusion}

This article has described a method of displaying bitmapped images under precise temporal control. The method makes use of color-lookup-table display hardware and the technique of color-table animation. The crucial problem to overcome using this technique is to find a way of allocating color-table indices to stimuli so that overlapping images can be drawn on the screen simultaneously while making efficient use of the pixel depth of screen memory. The present approach to this problem is to allocate indices to separate bit slices of screen memory when stimuli overlap and to allocate sequential indices when they do not. This method provides the ability to use commonly available video interfaces for a wide variety of research paradigms in which the temporal control of stimulus presentation is necessary.

\section{REFERENCES}

Costin, D. (1988). MacLab: A Macintosh system for psychology labs. Behavior Research Methods, Instruments, \& Computers, 20, 197-200. Dixon, P. (1984). A new technique for measuring word processing time in reading. Behavior Research Methods, Instruments, \& Computers, 16, 109-114.

FinLEY, G. P. (1989). Tachistoscopic software for the Hercules display controller. Behavior Research Methods, Instruments, \& Computers, 21, 387-390. 
Graves, R., Bradley, R. (1987). Millisecond interval timer and auditory reaction time programs for the IBM PC. Behavior Research Methods, Instruments, \& Computers, 19, 30-35.

HeATHCOTE, A. (1988). Screen control and timing routines for the IBM microcomputer family using a high-level language. Behavior Research Methods, Instruments, \& Computers, 20, 289-297.

Just, M. A., CArpenter, P. A., \& Wooley, J. D. (1982). Paradigms and processes in reading comprehension. Joumal of Experimental Psychology: General, 87, 329-354.

REED, A. V. (1979). Microcomputer display timing: Problems and solutions. Behavior Research Methods \& Instrumentation, 11, 572-576.

\section{NOTE}

1. Each entry in the color-lookup table specifies an RGB value, and each of the three RGB components has a 16-bit integer value. This yields a theoretical resolution of $2^{48}$, or roughly $10^{14}$, different colors. However, in practice, the number of physically different colors that can be shown on the screen is limited by the resolution of the digital-to-analog converters that generate the video signal.

(Manuscript received May 3, 1990; revision accepted for publication January 17, 1991.) 